\title{
Improvement in Acquired Bilateral Nevus of Ota-Like Macules with Picosecond-Domain Wavelength-Converted 595-nm Neodymium:Yttrium Aluminium Garnet Laser Treatment
}

Dae Suk Kim

Sung Bin Cho

Kangskin Sillim Dermatology Clinic, Seoul, Korea
Received December 9, 2016

Accepted December 16, 2016

\section{Correspondence}

Sung Bin Cho

Kangskin Sillim Dermatology Clinic, 339 Sillim-ro, Gwanak-gu, Seoul 08759, Korea

Tel.: +82-2-872-9007

Fax: +82-2-872-6226

E-mail: drsbcho@anaver.com

(C) Korean Society for Laser Medicine and Surgery

(c) This is an open access article distributed under the terms of the Creative Commons Attribution NonCommercial License (http://creativecommons.org/ licenses/by-nc/4.0) which permits unrestricted noncommercial use, distribution, and reproduction in any medium, provided the original work is properly cited.
The acquired bilateral nevus of Ota-like macules (ABNOM) is a common dermal pigmentary condition in Asians. Histologically, these lesions are similar to the nevus of Ota. However, ABNOM lesions differ in that melanocytes are located more superficially and clustered perivascularly. To treat $A B N O M$, various pigment-targeting $Q$-switched lasers have been used, although the treatment of ABNOM is more difficult than that of nevus of Ota. In this current report, we treated a patient with refractory ABNOM by targeting vessels in ABNOM lesions using a picoseconddomain wavelength-converted $595-\mathrm{nm}$ neodymium:yttrium aluminium garnet (Nd:YAG) laser. The patient showed clinical improvement in underlying vascular components and ABNOM lesions after two sessions of combined treatment with picosecond-domain 1,064-nm and wavelength-converted 595-nm Nd:YAG lasers. Accordingly, we suggest that a similar treatment may be promising as a therapeutic method for treating ABNOM.

\section{Key words}

Acquired bilateral nevus of Ota-like macules; Vascular component; Picosecond; Wavelength-converted 595 nm; Neodymium:Yttrium aluminium garnet 


\section{INTRODUCTION}

Acquired bilateral nevus of Ota-like macules (ABNOM), also called Hori's nevus, is common in Asian individuals. The dermal melanocytosis usually occurs bilaterally on the malar regions, temples, upper eyelids, and root and alae of the nose. ${ }^{1,2}$ The condition commonly presents in women after the third decade of life, appearing as discrete brown macules that become confluent slate-gray macules over time. ${ }^{2}$

Various treatment modalities have been introduced to treat ABNOM. Among them, Q-switched lasers, such as Q-switched ruby lasers, $Q$-switched neodymium:yttrium aluminium garnet (Nd:YAG) lasers, and Q-switched alexandrite lasers, have been most commonly used, showing varying responses and complication rates. Meanwhile, however, no report has described treating ABNOM with vascular targeting lasers. Vascular targeting lasers for melasma have been shown to elicit clinical improvements in the lesions and to decrease recurrence rates thereof. ${ }^{3,4}$ In this report, we present a case of refractory ABNOM that showed clinical improvement upon targeting underlying vessels with two sessions of combined picosecond 1,064-nm and wavelength-converted 595-nm Nd:YAG laser treatment.

\section{CASE REPORT}

A 62-year-old female patient presented with complex dyspigmentation on her face. She expressed a desire to undergo cosmetic treatment for darkened spots on her malar region in particular. She had previously been treated with several sessions of nanosecond-domain 1,064-nm Nd:YAG laser treatment, although with unsatisfactory clinical results. Physical examination revealed round, dark brown macules along the malar area bilaterally. Multiple dilated vessels were observed, especially along the dark brown macules. The patient was clinically diagnosed with ABNOM. We first treated the ABNOM lesions with five sessions of picosecond-domain 1,064-nm Nd:YAG laser (PICOPLUS; Lutronic Corp. Ltd., Goyang, Koreal treatment at the treatment settings of a $4-\mathrm{mm}$ spot size, $2.5 \mathrm{~J} / \mathrm{cm}^{2}$, and five passes at 2-4-week intervals. However, the lesions were refractory to the treatments.

After obtaining written informed consent, the patient was subsequently treated with combined picoseconddomain 1,064-nm and wavelength-converted 595-nm $\mathrm{Nd}$ :YAG laser (PICOPLUS) treatment for the pigmentary lesions of $\mathrm{ABNOM}$ and underlying vascular components, respectively, on her malar areas. Totally, two sessions of combination treatment were delivered at 2-week intervals with the treatment settings of a 1,064-nm wavelength, a spot size of $4 \mathrm{~mm}$, a fluence of $2.5 \mathrm{~J} / \mathrm{cm}^{2}$, and five passes on the pigmentary lesions and a 595 -nm converted wavelength, a spot size of $2 \mathrm{~mm}$, a fluence of $0.65 \mathrm{~J} / \mathrm{cm}^{2}$, and one pass along on the vascular lesions. Immediately after the picosecond-domain wavelength-converted 595-nm $\mathrm{Nd}$ :YAG laser treatment, a slight white frosting, edema, and erythema were seen on the treated lesions. The patient reported mild pain during the treatment, although no
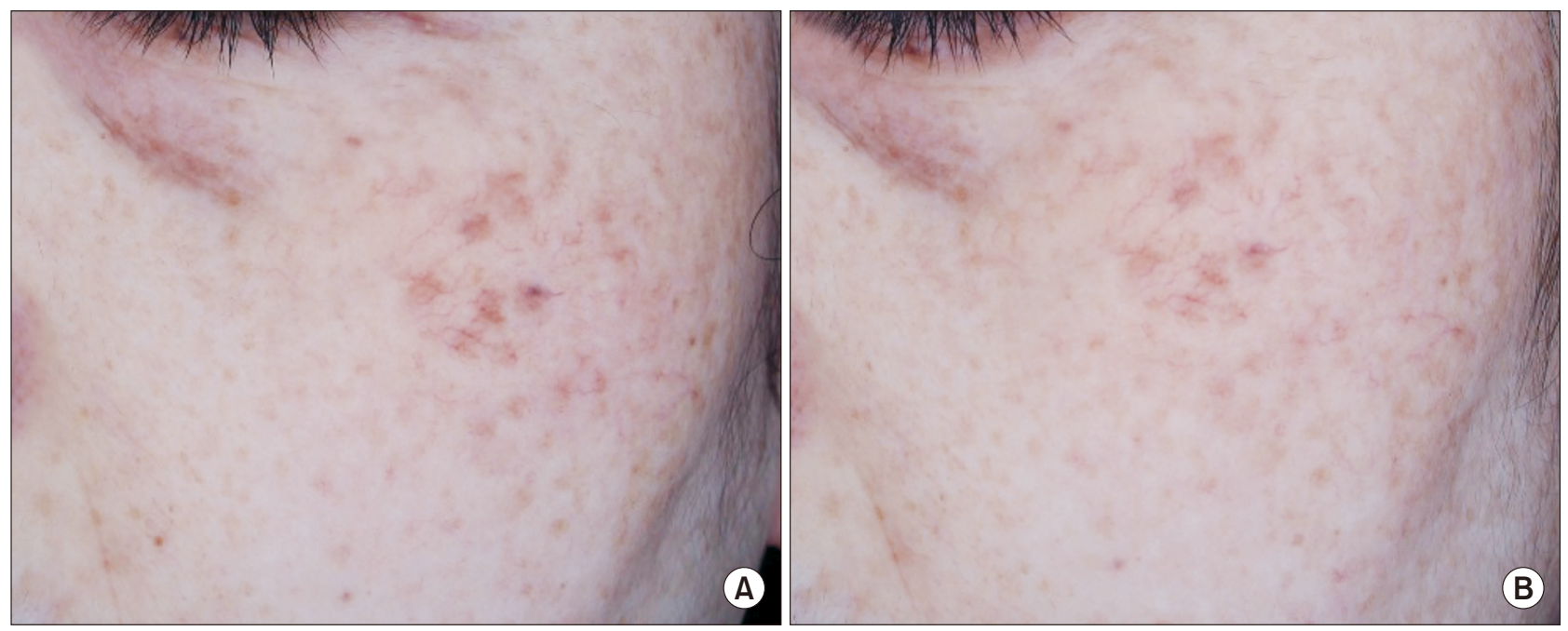

Fig. 1. Photographs taken under cross-polarized light exposure in a 62-year-old female patient with acquired bilateral nevus of Ota-like macules with adjacent vessels. Left side of her face (A) before the treatment and (B) 1 month after two sessions of combined picosecond-domain 1,064-nm and wavelength-converted 595-nm neodymium:yttrium aluminium garnet (Nd:YAG) laser treatment. 
immediate and late adverse events were recorded. Digital photographs were taken at baseline and 1 month after the final treatment (Fig. 1, 2). One month after the treatment, vessels along the malar regions had remarkably decreased in number and diameter, although complete eradication of vessels was not achieved. The ABNOM lesions along the targeted vessels, nonetheless, showed marked clinical improvement.

\section{DISCUSSION}

Nevus of Ota and ABNOM are histologically similar. Nevertheless, they can be distinguished by many clinical and histologic differences: ABNOM differs from nevus of Ota by its late onset, bilateral distribution, and lack of mucosal involvement. Although both diseases are most commonly treated with pigment targeting Q-switched lasers, it is well regarded that treatment of ABNOM is more difficult than that of nevus of Ota. ${ }^{5}$ In addition, postinflammatory hyperpigmentation (PIH) after laser treatment occurs more frequently in ABNOM than in nevus of Ota. Although a few hypotheses have been proposed to explain the higher tendency of PIH in ABNOM treatment, the precise causes of increased PIH after ABNOM treatment remain unknown. One study attributed the noted $\mathrm{PIH}$ to differences in histologic findings between nevus of Ota and ABNOM. ${ }^{5}$ Melanocytes of ABNOM are mostly located in the subpapillary dermis, clustered in groups, and dispersed perivascularly. Meanwhile, in nevus of Ota, melanocytes are distributed evenly throughout the papillary and reticular dermis and less commonly located ad- jacent to vessels, compared to ABNOM. ${ }^{5}$ The study noted a statistically significant correlation between the tendency of PIH after laser therapy and the number of melanocytes in the perivascular area in ABNOM. ${ }^{5}$ Lee, et al. concluded that indirect vessel injury during the destruction of perivascular clustering of melanocytes by laser therapy could be responsible for increased $\mathrm{PIH}^{5}$

Growing evidence suggests that increases in vascularization and vascular components in melasma lesions may play significant role in the pathogenesis of melasma. ${ }^{3}$ Indeed, 595-nm long-pulsed dye lasers targeting vascular components have been found to elicit added improvement and long-lasting effects in melasma treatments., Also, a recent study showed that endothelial cells promote skin pigmentation through endothelin receptor activation. ${ }^{6}$ Thus, interactions between vascular structures and perivascular melanocytes in ABNOM lesions could play important roles in hyperpigmentation. Accordingly, we speculated that vascular targeting therapy may exert beneficial effects when treating ABNOM.

In accordance with our speculation, the female patient described in this report showed improvement in ABNOM lesions with laser therapy targeting vascular structures. The patient was refractory to nanosecond-domain and picosecond-domain 1,064-nm Nd:YAG lasers treatments. We utilized the picosecond wavelength-converted 595-nm $\mathrm{Nd}$ :YAG laser at minimal laser fluence. By doing so, we found that the laser improved dilated vascular lesions to a similar degree as that achieved with pulsed dye lasers and cobber bromide lasers. Also, when treating vessels admixed with the hyperpigmented lesions of ABNOM, a
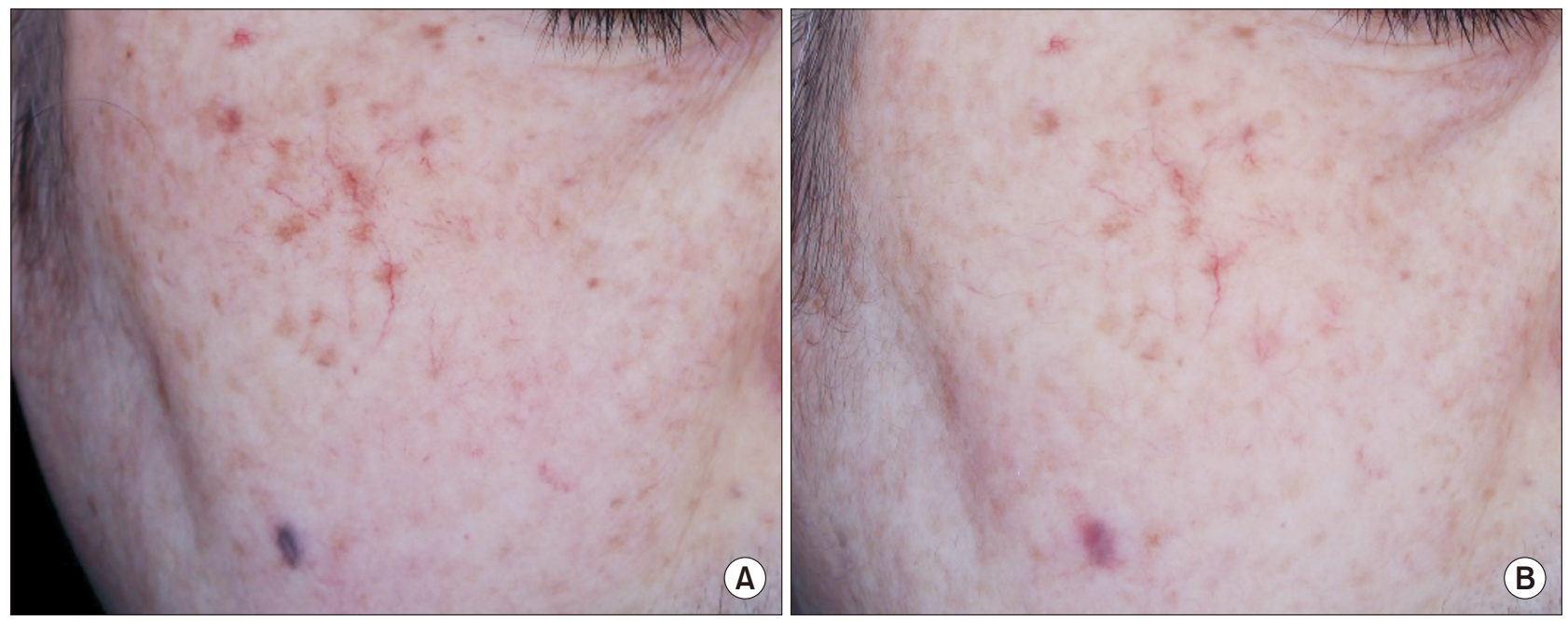

Fig. 2. Right side of the patient's face (A) before the treatment and (B) 1 month after two sessions of combined picosecond-domain 1,064-nm and wavelength-converted 595-nm Nd:YAG laser treatment. 
2-mm spot size allowed us to accurately aim at vascular structures while minimizing emission of the laser energy on adjacent skin. Nevertheless, whether picoseconddomain wavelength-converted 595-nm Nd:YAG lasers have vascular destructive or vascular modulative effects remains to be elucidated.

This is the first report to use a picosecond-domain, vascular targeting laser for the treatment of ABNOM. Accordingly, further investigation into the significance of vascular components in ABNOM lesions and more clinical studies on whether targeting vascular structures could be essential to ABNOM treatment are needed. Though there are various lasers to treat the vessels of the skin, we suggest that a picosecond 595-nm Nd:YAG laser could be a good option for treating ABNOM.

\section{REFERENCES}

1. Cho SB, Park SJ, Kim MJ, Bu TS. Treatment of acquired bilateral nevus of Ota-like macules (Hori's nevus) using 1064- nm Q-switched Nd:YAG laser with low fluence. Int J Dermatol 2009;:48:1308-12.

2. Park JM, Tsao H, Tsao S. Acquired bilateral nevus of Ota-like macules (Hori nevus): etiologic and therapeutic considerations. J Am Acad Dermatol 2009;61:88-93.

3. Geddes ER, Stout AB, Friedman PM. Long-pulsed dye laser of $595 \mathrm{~nm}$ in combination with pigment-specific modalities for a patient exhibiting increased vascularity within lesions of melasma. Dermatol Surg 2016;42:556-9.

4. Passeron T. Long-lasting effect of vascular targeted therapy of melasma. J Am Acad Dermatol 2013;69:e141-2.

5. Lee B, Kim YC, Kang WH, Lee ES. Comparison of characteristics of acquired bilateral nevus of Ota-like macules and nevus of Ota according to therapeutic outcome. J Korean Med Sci 2004; 19:554-9.

6. Regazzetti C, De Donatis GM, Ghorbel HH, Cardot-Leccia N, Ambrosetti D, Bahadoran P, et al. Endothelial cells promote pigmentation through endothelin receptor B activation. J Invest Dermatol 2015;135:3096-104. 